Research Paper

\title{
Up-Regulated AKR1C2 is correlated with favorable prognosis in thyroid carcinoma
}

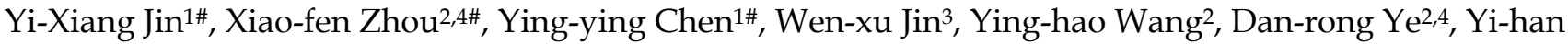

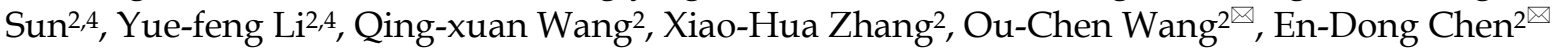 \\ 1. Department of Colorectal and Anal Surgery, The First Affiliated Hospital of Wenzhou Medical University, Wenzhou, Zhejiang Province, 325000, China \\ 2. Department of Thyroid and Breast Surgery, The First Affiliated Hospital of Wenzhou Medical University, Wenzhou, Zhejiang Province, 325000, China \\ 3. Department of Vascular Surgery, The First Affiliated Hospital of Wenzhou Medical University, Wenzhou, Zhejiang Province, 325000, China \\ 4. Wenzhou Medical University, Wenzhou, Zhejiang Province, 325000, China \\ \# These authors contributed equally to this work.
}

$\square$ Corresponding authors: Drs. En-Dong Chen and Ou-Chen Wang, Department of Thyroid and Breast Surgery, The First Affiliated Hospital of Wenzhou Medical University, Wenzhou, Zhejiang, China. Tel/fax: +86 0577 55579462; E-mail: chenendong.oncology@gmail.com

(C) Ivyspring International Publisher. This is an open access article distributed under the terms of the Creative Commons Attribution (CC BY-NC) license (https://creativecommons.org/licenses/by-nc/4.0/). See http://ivyspring.com/terms for full terms and conditions.

Received: 2018.07.07; Accepted: 2019.04.27; Published: 2019.06.09

\begin{abstract}
Purpose: Aldo-keto reductase family 1, member C2 (AKR1C2) gene encodes for a member of the AKR superfamily and participates in the metabolism of various drugs. Moreover, tumor and normal tissues exhibit an evident difference in the expression level of this gene.

Methods: We downloaded and analyzed AKRIC2 expression level and the data consisting of the clinicopathological features of 490 papillary thyroid carcinoma (PTC) tumor tissues and 59 normal thyroid tissues from The Cancer Genome Atlas (TCGA) cohort. Diverse statistical methods, such Chi-square test, univariate and multivariate Cox regression analyses, and Kaplan-Meier survival curves were used. We down-/up-regulated the expression of AKR1C2 and explored its specific role in thyroid cancer cell lines by utilizing the si-RNA and plasmid.

Results: We divided all patients who were collected in TCGA data sets into under-expressed $(n=245)$ and over-expressed groups $(n=245)$. We subsequently analyzed the data and obtained the following findings: (a) AKRIC2 is down-regulated in papillary thyroid carcinoma (PTC) $(p<0.001)$, (b) Kaplan-Meier result revealed that high expression level of AKRIC2 are correlated with favorable survival in PTC $(p=0.043)$, and (c) factors independently associated with recurrence-free survival are AKR1C2 expression (hazard ratio (HR 0.819) and American Joint Committee on Cancer (AJCC) stage (HR 1.534). We also analysed the relationship between $A K R 1 C 2$ expression and clinicopathological features in the validated cohort. AKR12C under-expression correlated with lymph node metastasis $(p=0.009)$ and AJCC stage $(p=0.001)$ which might indicate AKR 12C as a prognostic factor in PTC. The cell line experiment results showed that the knockdown and overexpression of AKR IC2 significantly enhance and weaken the abilities of migration and invasion in papillary thyroid carcinoma cell.

Conclusion: Our results indicated that AKR1C2 exerts inhibitory effects on PTC oncogenesis and elevated $A K R 1 C 2$ expression is associated with the favorable prognostic factors and recurrence free survival.
\end{abstract}

Key words: AKR1C2, Papillary thyroid carcinoma, Prognosis, Expression

\section{Introduction}

Thyroid carcinoma is the most prevalent malignant tumor in the endocrine system [1]. The number of thyroid carcinoma incidences has continuously gone up worldwide in the past few decades. In America, the incidence of thyroid carcinoma increased stably after 1990s. And the mortality of thyroid carcinoma has stably remained over the 35-year period [2-4]. The same trend was observed in China: thyroid cancer was ranked as fourth most common cancer among women in urban Beijing in 2012 [5].

Most patients with PTC exhibited favorable outcomes, and 30-year survival rates were recorded in more than $90 \%$ of patients who underwent routine 
treatment via thyroidectomy [6, 7]. However, regardless of the outcome, some patients may still suffer from tumor aggressiveness, additionally, some PTCs progress into radioiodine-refractory disease and evolve into metastasis and/or recurrence (about 20\% after a 10-year follow-up) [8]. Therefore, early diagnosis and treatment plan should be developed to improve the prognosis and life quality of patients. Current research on molecular mechanisms of thyroid oncogene or oncosuppressor provided abundant knowledge about PTC. Among these mechanisms, the activation of the mitogen-activated protein kinase (MAPK) signaling pathway is the most common; it results from the mutations of the oncogene $\mathrm{BRAF}$; meanwhile, the BRAF mutation prevalence of PTC is approximately $45 \%$ [9-11]. Fortunately, cumulative efforts over many decades have resulted in the discovery of promising biomarkers and therapeutic targets for early diagnosis and treatment of PTC.

The aldo-keto reductase (AKR) superfamily of enzymes is critical for drug metabolism and toxin detoxification in the human body; this superfamily catalyzes the carbonyl groups to product primary and secondary alcohols with a widespread substrates and ketones into corresponding alcohol compounds[12, 13]. However, the role of AKR family 1 member $C 2$ (AKR1C2) in a various cancer types is rarely investigated. In esophageal squamous cell carcinoma, overexpressed AKR1C2 increases the sensitivity of cells to ethyl-3,4-dihydroxybenzoate, otherwise known as protocatechuic acid ethyl ester, indicating that AKR1C2 exerts a suppressive effect on esophageal squamous cell carcinomas[12]. AKR1C2 expression exhibits a positive correlation with favorable tumor characteristics and long survival in primary breast cancer patients [14]. Differently, in liver cancer, AKR1C2 was proved to be a positive regulator in promoting metastasis [15]. As an enzyme that is critical for drug metabolism and toxin detoxification, AKR1C2 exerts inhibitory effects on sensitivity to anticancer drug resistance; these anticancer drugs include cis-diamminedichloroplatinum (II) and 5-fluorouracil [16, 17]. Nevertheless, only a few studies focused on thyroid, none of them concerned about thyroid cancer. Moreover, the relationship between AKR1C2 expression and prognosis remains unclear.

In present study, we downloaded and re-analyzed 490 The Cancer Genome Atlas (TCGA) [18] patients' AKR1C2 mRNA expression levels and clinical data. To exclude the influence of differences between individuals, we also enrolled 38 pairs of tumor and matched adjacent non-cancerous tissues to analyze AKR1C2 mRNA expression levels and clinical features. Meanwhile, in vitro experiments were performed to explore the specific effect of AKR1C2 in thyroid carcinoma.

The aims of our research are as follows: (a) to detect AKR1C2 expression in PTC patients, (b) to figure out the connection between AKR1C2 expression and clinical characteristics of PTCs, and (c) to further examine AKR1C2 functions and its effect on PTC prognosis.

\section{Materials and methods}

\section{Clinical samples}

During the initial surgery, the selection of 38 primary PTCs and corresponding adjacent normal tissues were ratified by the Ethics Committee of the First Affiliated Hospital of Wenzhou Medical University. Primary PTC tissues were surgically resected, and then quickly frosted in liquid nitrogen, and subsequently, reserved in a freezer at eighty degrees below zero. A retrospective analysis of the histopathological sections of all cases was conducted by two experienced pathologists to determine the diagnosis of histomorphology of the tumor.

The Illumina HiSeq 2000 Sequencing platform by TCGA genome characterization center was used for the measurement of gene expression profile. From TCGA data coordination center in March 2016 access, the date of AKR1C2 Level 3 was downloaded. This dataset indicates the transcriptional estimates of the gene level, as in $\log 2(x+1)$ transformed RSEM normalized count. In the format of 'Biotab', and from the portal of TCGA, we downloaded TCGA clinical data and re-analyzed 490 TCGA patient clinical data that had the relapse free survival records. In term of median of AKR1C2 mRNA expression levels, we divided those 490 PTC patients into under-expressed group $(n=245)$ and over-expressed groups $(n=245)$. The available clinical data include the following categories: histological type, tumor size, unilateral or bilateral type, presence of thyroiditis, multifocal or unifocal carcinoma, extrathyroidal invasion, lymph node metastasis, metastasis and American Joint Committee on Cancer (AJCC) stage. Available population data are as follows: age at diagnosis, gender, and time of follow-up.

\section{Cell lines and cell culture}

TPC-1 and BCPAP, two types of thyroid cancer cell lines, that were obtained from Mingzhao Xing, a Professor in the Johns Hopkins University School of Medicine (Baltimore, MA, USA) were cultured in a RPMI1640 (Gibco, C11875500BT) culture medium that contained with $10 \%$ fetal bovine serum (FBS) (Gibco, 10099141), $1 \times$ sodium pyruvate (Gibco, 11360-070) as well as $1 \times$ MEM nonessential amino acids (Gibco, 11140-050). Thyroid cancer cell line KTC-1 (from Stem 
Cell Bank, Chinese Academy of Sciences) was cultured in a RPMI1640 culture medium that contained with $10 \% \mathrm{FBS}$ together with $1 \times$ MEM nonessential amino acids. The aforementioned three cell lines were cultured in an incubator with $5 \% \mathrm{CO}_{2}$ at $37^{\circ} \mathrm{C}$ with a humidified atmosphere.

\section{RNA extraction and real-time qRT-PCR}

ReverTra Ace ${ }^{\circledR}$ qPCR RT Kit (Toyobo, Co., Ltd., Osaka, Japan) was applied for the determination of the cDNA. The quantitative Real-time Polymerase Chain Reaction (Real-time qRT-PCR) was carried out with the application of THUNDERBIRD SYBR qPCR Mix (Toyobo, Co., Ltd., Osaka, Japan) in the ABI 7500 Sequence Detection System (Applied Biosystems, Foster City, CA, USA). The forward and reverse primer sequence of AKR1C2 for PCR was 5'-GAC AGGCATGAAGTGACCATC-3' and 5'-GAAGAAAC ATTTGCTAACCAGG-3', respectively. Each sample was tested for three times. Glyceraldehyde phosphate dehydrogenase (GAPDH) was selected as an internal control.

\section{Protein extraction and Western blot analysis}

The lysis of collected cells was performed with radio-immunoprecipitation assay (RIPA) lysis buffer, which was purchased from Beyotime Institute of Biotechnology, Shanghai, China. Approximately 20 $\mu \mathrm{g}$, an equal volume of protein, was separated using a $10 \%$ SDS-PAGE buffer and subsequently, electroblotted onto the polyvinylidene fluoride (PVDF) membrane. The PVDF membrane, after which was blocked with 5\% condensed skimmed milk (BD,Difco ${ }^{\mathrm{TM}}$ Skim Milk, 232100), was incubated with anti-AKR1C2 antibody (Abcam, Boston, MA, USA). Subsequently, the incubation of the above mentioned membrane was performed with horseradish peroxidase HPR-labeled secondary antibody IgG (Abcam, Boston, MA, USA) for 1.5 hours at room temperature after which were washed with tris-buffered saline and Tween 20 (TBST) for thrice. $\beta$-actin, which was used for internal control, was detected by using an anti- $\beta$-actin antibody (Abcam, Boston, MA, USA).

\section{RNA interference and plasmid preparation}

Small interfering RNA (si-RNA) targeting AKR1C2 and negative control si-RNA (si-NC) were purchased from Gene Pharma (Shanghai, China) for the knockdown of gene expression. The sequences of the si-RNA were as follows: ARK1C2, forward 5'-GAGAUUCCUUCUCAAGCCATT-3' and reverse 5'-UGGCUUGAGAAGGAAUCUCTT-3'. The cells were transfected at $50 \%-60 \%$ confluence using Lipofectamine RNAiMAX (Invitrogen, Grand IsLand, NY). Human AKR1C2 cDNA ORF clone (cat.
RC213538) and the empty control pCMV6- Entry vector (cat. PS100001) were purchased from OriGene (Rockville, MD, USA). Human PTC cell lines TPC-1, BCPAP and KTC-1 cells were transiently transfected with either the AKR1C2 cDNA ORF clone or pCMV6Entry vector using Lipofectamine 3000 (Invitrogen, L3000015). Those treated cells were harvested $48 \mathrm{~h}$ after transfection for RNA expression analysis and subsequent assays. All knockdown and overexpression experiment were performed at least three times independently for each cell line.

\section{Migration and invasion assays}

The transwell cell culture chambers and BioCoat ${ }^{\mathrm{TM}}$ Matrigel Invasion Chamber 24-Well Plate 8.0 Micron (Corning Costar Corp, Cambridge, MA, USA) were utilized to perform transwell migration and invasion assays, respectively. The three transfected cell lines were seeded in the apical chamber of transwell chamber, and migration and invasion assays was conducted in the basolateral chamber that imbued with culture medium, which was added with $10 \%$ and $20 \%$ FBS, separately. The cells were wiped off if they did not traverse the filter after 24h. Migrating cells, which located on the opposite side of the filter, were immobilized with four percent PFA (Sigma-Aldrich, St Louis, MO, USA) for 30 minutes, dyed with 0.01 percent crystal violet for 60 minutes, photographed, and finally, counted under a light microscope.

\section{Statistical Analysis}

Data that obey the normal distribution were showed in the form of mean and standard variation, and the results were analyzed by t-test. The enumeration data were expressed in the form of percentage and were analyzed by Chi-square test or deemed appropriate, Fisher's exact test results. Factors independently related to the recurrence-free survival were tested using Cox regression analysis. Kaplan-Meier method was utilized for the estimation of 6 to 5423 days' recurrence-free survival (RFS) curve, and differences in RFS curve between or among patient groups were assessed using the log rank-test. The influences of covariates of interest on recurrence-free survival were assessed by multicovariate Cox's proportional hazards regression model. Kaplan-Meier survival curves were used to indicate the prognosis of different expression level groups. The value of $p$ no more than 0.05 were deemed to have significant difference. Data were analyzed and power analysis was carried out with 20.0 SPSS software (IBM Corp, New York, USA). 


\section{Results}

\section{AKR1C2 is down-regulated in PTC}

AKR1C2 expression is detected in 490 PTC tumor tissues and 59 normal thyroid tissues from TCGA cohort and 38 tissue samples contained PTC tumor samples and matched adjacent noncancerous tissues from validation cohort. As shown in Fig. 1a and $1 b$, the AKR1C2 expression in tumor tissues is dramatically lower than that in normal thyroid tissues $(4.19 \pm 1.81$ vs. $7.01 \pm 0.95, p<0.001$ in TCGA cohort and $1.35 \pm 2.34$ vs. $7.07 \pm 9.18, p<0.001$ in validation cohort). The potential tumor-suppressive role of AKR1C2 in PTC oncogenesis is implied by the different levels of AKR1C2 expression between normal and tumor tissues.

\section{The clinicopathological characteristics of low AKRIC expression in PTC}

To investigate whether or not AKR1C2 expression is associated with the tumorigenesis and progression of PTC, we analyzed the association between the AKR12C and the clinicopathological features of PTC. As shown in Table 1, the analysis revealed big differences in histological type $(p=0.013)$, tumor size $(p=0.049)$, unilateral or bilateral $(p=0.016)$ and unifocal or multifocal $(p=0.044)$ in TCGA cohort. In the validated cohort (Table 2), the analysis revealed big differences in unilateral or bilateral $(p=0.048)$, lymph node metastasis $(p=0.009)$, AJCC disease stage $(p=0.001)$. Nevertheless, no relationships were found between AKR1C2 expression and age, gender, size and extrathyroidal invasion.

Table 1. The relationship between AKRIC2 expression and clinicopathological characteristics in TCGA cohort

\begin{tabular}{|c|c|c|c|}
\hline \multirow[t]{2}{*}{ Characteristics } & \multicolumn{3}{|c|}{$\begin{array}{l}\text { Expression of AKR1C2, Number (\%) } \\
\text { (on the basis of the median value) }\end{array}$} \\
\hline & LOW (n=245) & HIGH $(n=245)$ & $p$-value \\
\hline \multicolumn{4}{|l|}{ Age at diagnosis, $y$} \\
\hline Mean \pm SD & $47.249 \pm 15.58$ & $47.282 \pm 16.01$ & 0.817 \\
\hline$<45 y$ & $109(44.49 \%)$ & $113(46.12 \%)$ & 0.969 \\
\hline$\geq 45 y$ & $136(55.51 \%)$ & $132(53.88 \%)$ & \\
\hline Gender & & & 0.054 \\
\hline Female & $188(76.73 \%)$ & $169(68.98 \%)$ & \\
\hline Male & $57(23.27 \%)$ & $76(31.02 \%)$ & \\
\hline Histological type & & & $0.013^{*}$ \\
\hline Classical & $179(73.06 \%)$ & $169(68.98 \%)$ & \\
\hline Follicular & $40(16.33 \%)$ & $59(24.0 \%)$ & \\
\hline Tall Cell & $24(9.80 \%)$ & $11(4.49 \%)$ & \\
\hline Other specify & $2(0.82 \%)$ & $6(2.45 \%)$ & \\
\hline \multicolumn{4}{|l|}{ Tumor size in $\mathrm{cm}$} \\
\hline Mean \pm SD & $2.98 \pm 1.64$ & $2.79 \pm 1.66$ & 0.21 \\
\hline$\leq 2 \mathrm{~cm}$ & $74(30.20 \%)$ & $91(37.14 \%)$ & $0.049^{*}$ \\
\hline$>2 \mathrm{~cm}$ & $159(64.90 \%)$ & $133(54.29 \%)$ & \\
\hline T stage & & & 0.072 \\
\hline $\mathrm{T} 1$ & $61(24.90 \%)$ & $79(32.24 \%)$ & \\
\hline$>\mathrm{T} 1$ & $183(74.69 \%)$ & $165(67.35 \%)$ & \\
\hline Unilateral or Bilateral & & & $0.016^{*}$ \\
\hline Unilateral & $210(85.71 \%)$ & $190(77.55 \%)$ & \\
\hline Bilateral & $32(13.06 \%)$ & $52(21.22 \%)$ & \\
\hline
\end{tabular}

\begin{tabular}{llcc}
\hline Characteristics & \multicolumn{3}{l}{$\begin{array}{l}\text { Expression of AKR1C2, Number (\%) } \\
\text { (on the basis of the median value) }\end{array}$} \\
\hline $\begin{array}{l}\text { Thyroiditis } \\
\text { Multifocal or Unifocal }\end{array}$ & $36(14.69 \%)$ & $33(13.47 \%)$ & 0.697 \\
$\begin{array}{l}\text { Multifocal } \\
\text { Unifocal }\end{array}$ & $99(40.41 \%)$ & $121(49.39 \%)$ & $0.044^{*}$ \\
Extrathyroidal invasion & $141(57.55 \%)$ & $119(48.57 \%)$ & \\
YES & & & 0.085 \\
NO & $83(33.88 \%)$ & $65(26.53 \%)$ & \\
Lymph node metastasis & $154(62.86 \%)$ & $170(69.39 \%)$ & 0.115 \\
YES & $117(47.76 \%)$ & $98(40.00 \%)$ & \\
NO & $106(43.27 \%)$ & $120(48.98 \%)$ & 0.055 \\
Metastasis & & & \\
M0 & $130(53.06 \%)$ & $148(60.41 \%)$ & \\
M1 & $7(2.86 \%)$ & $1(0.41 \%)$ & 0.422 \\
AJCC disease stage & & & \\
I+II & & \\
III+IV & $160(65.31 \%)$ & $169(68.98 \%)$ & \\
\hline * $p$-value $<0.05$. & $84(34.29 \%)$ & $76(31.02 \%)$ & \\
& &
\end{tabular}

Table 2. The relationship between AKR1C2 expression and clinicopathological characteristics in validated cohort

\begin{tabular}{|c|c|c|c|}
\hline \multirow[t]{2}{*}{ Characteristics } & \multicolumn{2}{|c|}{$\begin{array}{l}\text { Expression of AKR1C2, Number (\%) (on } \\
\text { the basis of the median value) }\end{array}$} & \multirow[b]{2}{*}{$p$-value } \\
\hline & LOW $(n=19)$ & HIGH (n=19) & \\
\hline \multicolumn{4}{|l|}{ Age at diagnosis, $y$} \\
\hline Mean \pm SD & $45.79 \pm 6.63$ & $45.79 \pm 11.43$ & 1 \\
\hline$<45 y$ & $7(36.84 \%)$ & $8(42.11 \%)$ & 0.74 \\
\hline$\geq 45 y$ & $12(63.16 \%)$ & $11(57.89 \%)$ & \\
\hline Gender & & & 0.074 \\
\hline Female & $16(84.21 \%)$ & $11(57.89 \%)$ & \\
\hline Male & $3(15.79 \%)$ & $8(42.11 \%)$ & \\
\hline \multicolumn{4}{|l|}{ Tumor size in $\mathrm{cm}$} \\
\hline Mean \pm SD & $13.95 \pm 6.753$ & $18.47 \pm 8.853$ & 0.085 \\
\hline$\leq 1 \mathrm{~cm}$ & $4(21.05 \%)$ & $3(15.79 \%)$ & 0.676 \\
\hline$>1 \mathrm{~cm}$ & $15(78.95 \%)$ & $16(84.21 \%)$ & \\
\hline Unilateral or Bilateral & & & $0.048^{*}$ \\
\hline Unilateral & $11(57.89 \%)$ & $7(36.84 \%)$ & \\
\hline Bilateral & $8(42.11 \%)$ & $12(63.16 \%)$ & \\
\hline Extrathyroidal invasion & & & 0.311 \\
\hline YES & $18(94.74 \%)$ & $19(100 \%)$ & \\
\hline NO & $1(5.26 \%)$ & $0(0 \%)$ & \\
\hline Lymph node metastasis & & & $0.009^{*}$ \\
\hline YES & $12(63.16 \%)$ & $4(21.05 \%)$ & \\
\hline NO & $7(36.84 \%)$ & $15(78.94 \%)$ & \\
\hline AJCC disease stage & & & $0.001^{*}$ \\
\hline I & $8(42.11 \%)$ & $0(0 \%)$ & \\
\hline II & $1(5.26 \%)$ & $2(10.53 \%)$ & \\
\hline III & $10(52.63 \%)$ & $9(47.36 . \%)$ & \\
\hline IV & $0(0 \%)$ & $8(42.51 \%)$ & \\
\hline
\end{tabular}

\section{Relationship between AKR1C2 expression and recurrence-free survival}

After analyzing the clinical feature data, we also determined the relationship between clinicopathological characteristics and RFS. The factors highly connected with RFS were determined through univariate Cox regression analysis. As shown in Table 3, the results included the following factors: tumor stage (hazard ratio (HR) 1.588; 95\% confidence interval (CI), 1.110-2.272; $p=0.011)$, metastasis (HR, 8.995; 95\% CI, 2.596-30.897, $p=0.001)$, AJCC stage $(\mathrm{HR}, 1.528 ; 95 \% \mathrm{CI}, 1.167-2.001 ; p=0.002)$, and AKR1C2 expression (HR, 0.824; 95\% CI, 0.681-0.998; $p$ 
$=0.047)$. To determine the number of independent prognostic factors, multivariate Cox regression analysis was carried out. The results revealed the following factors to be independently associated with RFS (Table 4): AKR1C2 expression (HR, 0.819; 95\% CI, $0.677-0.992 ; p=0.041)$ and AJCC stage (HR, $1.534 ; 95 \%$ CI, 1.172-2.009; $p=0.002$ ). Finally, to prove whether AKR1C2 expression levels are correlated with PTC prognosis, we analyzed the follow-up data in TCGA cohort. The medium follow-up time is 1156 days (ranged from 6 days to 5423 days). In the Kaplan-Meier survival curve (Fig. 1c), remarkable difference was observed between the RFS rates of the under-expressed group $(\mathrm{n}=245)$ and over-expressed groups $(n=245)$. The 1000 and 2000 days of RFS rates in the overexpressed group are $95 \%$ and $90 \%$, respectively, all of which are higher than the 1000 and 2000 days of RFS rates, which are $90 \%$ and $82 \%$, respectively, in the under-expressed group $(p=0.043)$. The univariate Cox $\mathrm{PH}$ (Cox proportional hazard model) analysis and Multivariate Cox $\mathrm{PH}$ model analysis revealed high AKR1C2 expression is strongly correlated with favorable patient survival $(p=0.047$; HR, 0.509; Fig. $1 \mathrm{~d}$ and $p=0.050$; HR, 0.514; Fig. 1e respectively). Therefore, we assumed that AKR1C2 expression might be an independent protective prognosis factor given that its expression shows HR $<1$ and $p<0.05$.

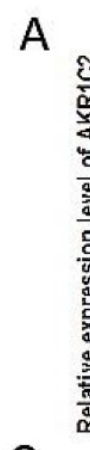

C
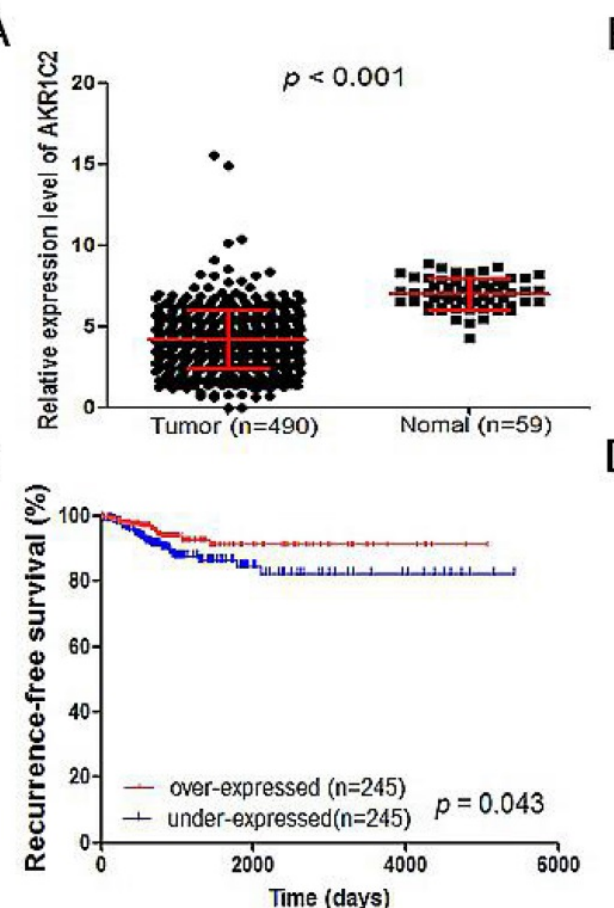
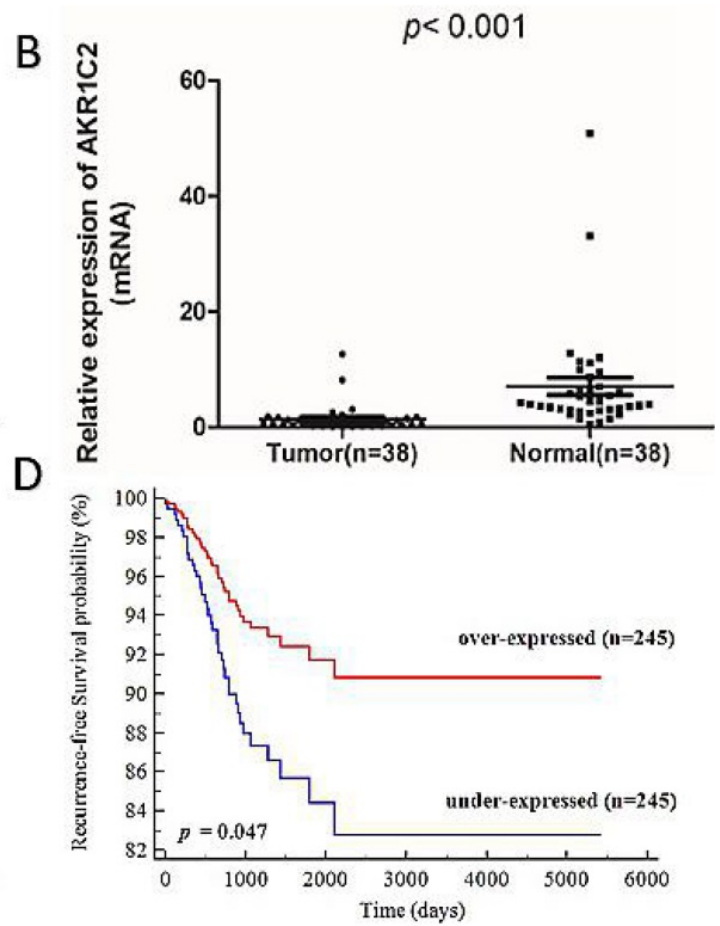

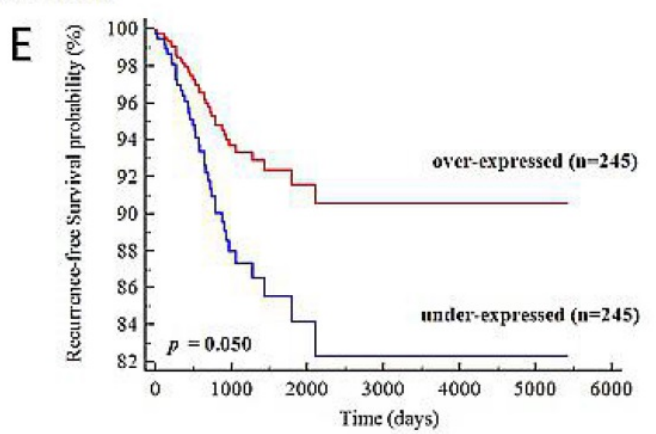

Figure 1. Gene AKRIC2 was remarkly downregulated in thyroid cancer tissues compared with adjacent normal thyroid tissues in both validation cohort and TCGA cohort. Kaplan-Meier analysis for the recurrence-free survival of PTC patients with different expression level of AKRIC2 in TCGA cohort. Notes: (a) Not in accordance with normal distribution, the Mann-Whitney $U$ test was used to evaluate the expression difference between PTC tumors tissues and adjacent normal tissues in the TCGA cohort. The TCGA cohort included 490 tumor tissues samples and 59 normal tissue samples $(p<0.001)$. (b) Not in accordance with normal distribution, analysis of expression of AKRIC2 was done using the Mann-Whitney $U$ test in the validation cohort. Gene AKRIC2 expression was measured by qRT-PCR in 38 paired PTC tissues and adjacent normal tissues $(p<0.001)$. (c) Kaplan-Meier curve analysis for the RFS of papillary thyroid carcinoma patients with different expression of AKR1C2 in TCGA cohort. Patients with low AKR1C2 expression had a shorter RFS than high AKRIC2 expression. (d) Unadjusted survival between the low and high AKRIC2 groups was compared using univariate Cox PH modeling (categorical variable). (e) Adjusted survival with respect to AKRIC2 expression was assessed using a multivariate Cox PH approach. Survival adjusted for patient age at diagnosis, gender and AJCC stage (categorical variable). 


\section{AKR1C2 knockdown potentiates the capacities of migration and invasion in PTC cells}

To explore the function of AKR1C2 in thyroid cancer, with the use of si-RNA, we downregulated the expression levels of AKR1C2. In Fig. 2a and 2b, the AKR1C2 expression descended effectively in all three PTC cell lines TPC-1, BCPAP and KTC-1 $(p<0.001)$, as confirmed by qRT-PCR and Western blot. Afterwards, given the expression of AKR1C2 is associated with clinicopathological characteristics in TCGA cohort, we investigated whether AKR1C2 could influence metastasis in thyroid cancer cell lines. As shown in Fig. $2 \mathrm{c}$ to $2 \mathrm{f}$, Transwell migration and Transwell invasion assays were applied. Results turned out that AKR1C2 knockdown significantly enhances the capacities of migration and invasion in TPC-1, BCPAP and KTC-1 $(p<0.05)$.

Table 3. Univariate cox regression analysis of recurrence-free survival.

\begin{tabular}{llll}
\hline Characteristics & HR & $95.0 \%$ CI & $p$-value \\
\hline Age & 1.013 & $0.993-1.033$ & 0.194 \\
Gender & 1.460 & $0.750-2.845$ & 0.266 \\
Histological type & 1.150 & $0.749-1.766$ & 0.523 \\
Tumor size & 1.154 & $0.970-1.375$ & 0.107 \\
T stage & 1.588 & $1.110-2.272$ & $0.011^{*}$ \\
Unilateral or Bilateral & 1.485 & $0.679-3.250$ & 0.322 \\
Multifocal or Unifocal & 1.267 & $0.672-2.389$ & 0.465 \\
Thyroiditis & 1.147 & $0.477-2.757$ & 0.760 \\
Extra thyroidal invasion & 1.332 & $0.694-2.557$ & 0.388 \\
Lymph node metastasis & 1.454 & $0.749-2.821$ & 0.268 \\
Metastasis & 8.955 & $2.596-30.897$ & $0.001^{*}$ \\
AJCC stage & 1.528 & $1.167-2.001$ & $0.002^{*}$ \\
AKR1C2 expression (continuous variable) & 0.824 & $0.681-0.998$ & $0.047^{*}$ \\
\hline
\end{tabular}

* $p$-value $<0.05$.
A

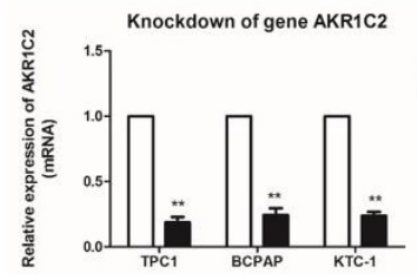

C

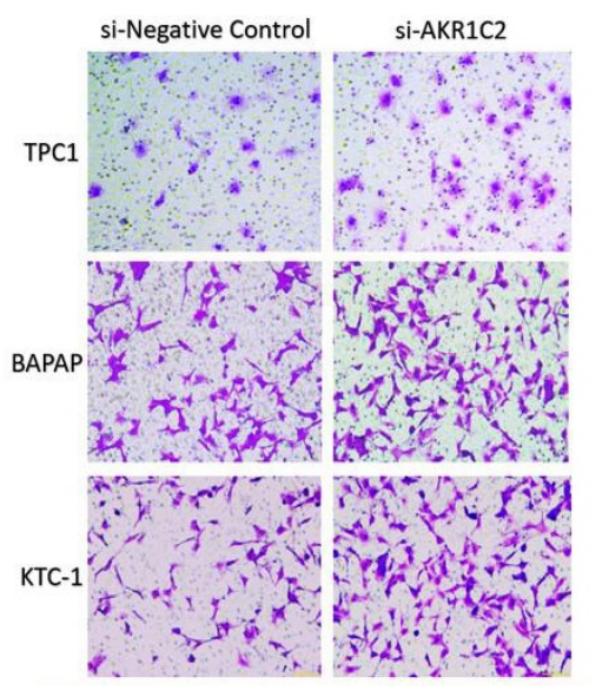

D

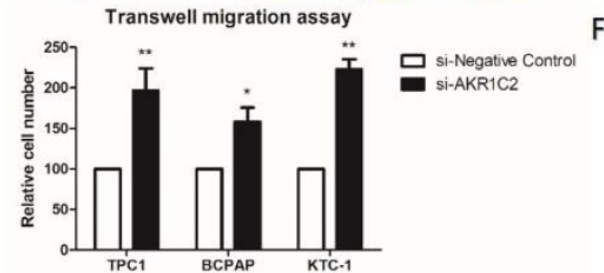

口 si-Negative Control si-Negative Cont-AKR1C2

$E$

$\mathrm{F}$
B

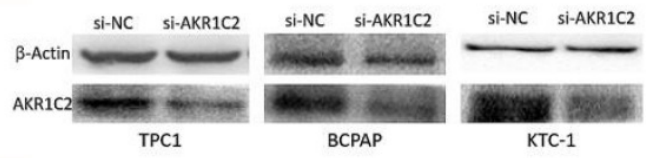

si-Negative Control Si-AKR1C2
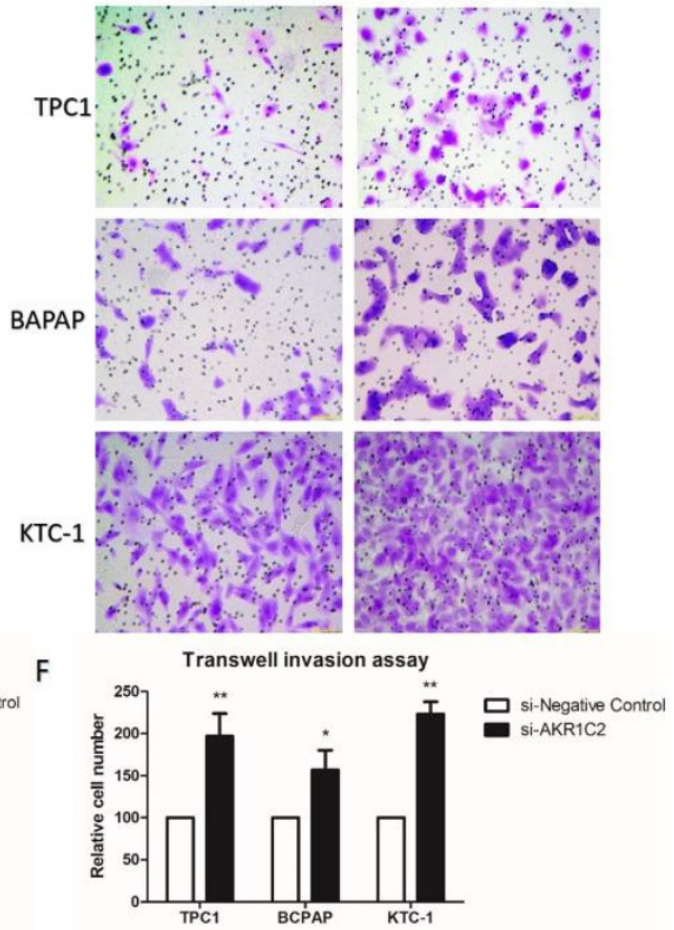

Figure 2. The impact of knockdown of AKR1C2 on cell migration and invasion of TPC-1, BCPAP as well as KTC-1 PTC cell lines. Notes (a) qRT-PCR was applied for the confirmation of the knockdown of AKR1C2. Transfected by targeted si-AKR1C2, the level of mRNA expression of AKR1C2 was remarkably decreased in TPC-1, BCPAP and KTC-1 cell lines in contrast to si-NC (negative control). (b) Western blot was used for confirming the knockdown of AKR IC2. Transfected by targeted si-AKR1C2, the level of protein expression of AKR1C2 was remarkably downregulated in TPC-1, BCPAP and KTC-1 cell lines in contrast to si-NC (negative control). (c) As for migration assays, we found that compared with the cells transfected by si-NC, the migrating cells transfected by si-AKR1C2 were much more in BCPAP, TPC1, and KTC-1 cell lines. (d) The number of the migrating cell by relative quantification. (e): With respect to invasion assays, we found that the invading cells transfected by si-AKR1C2 were much more in BCPAP, TPC1, and KTC-1 cell lines compared with the cells transfected by si-NC. (f) The number of the invading cell by relative quantification. The Columns refers to the average number of invading cell from no less than three experiments; and the little vertical bars, which located at the top end of the columns, corresponds to standard deviation (SD). $* p<0.1, * * p<0.05, * * * p<0.01$ using student's t-test. 
A

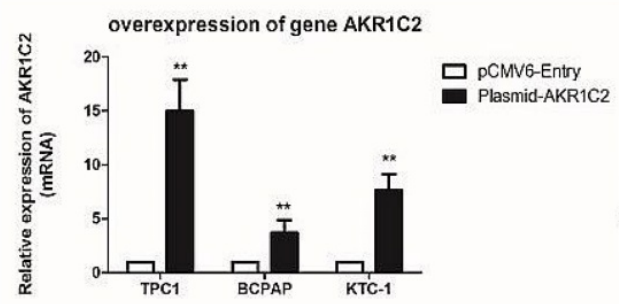

B

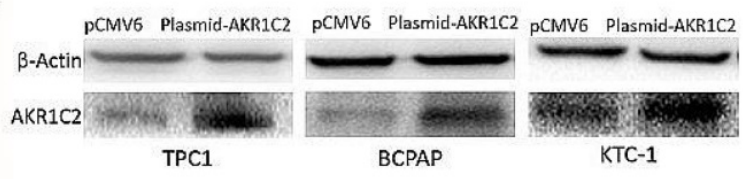

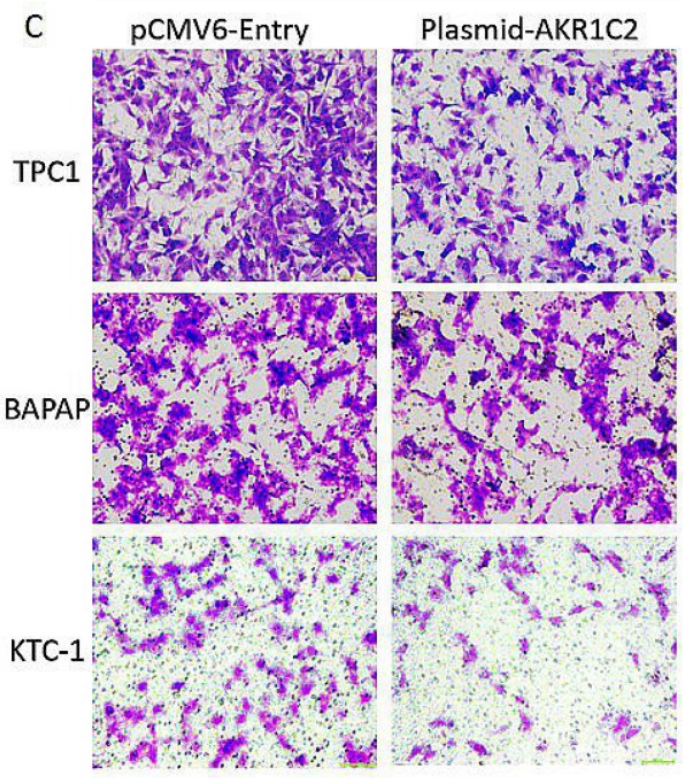

D

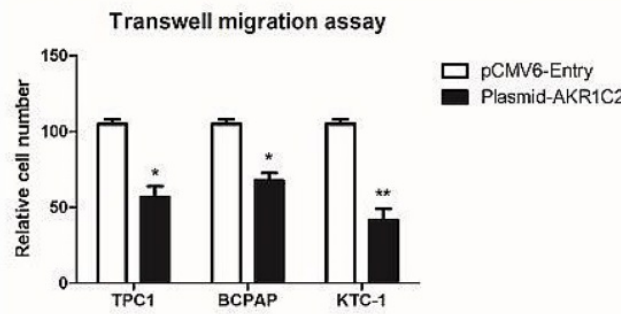

Plasmid-AKR1C2
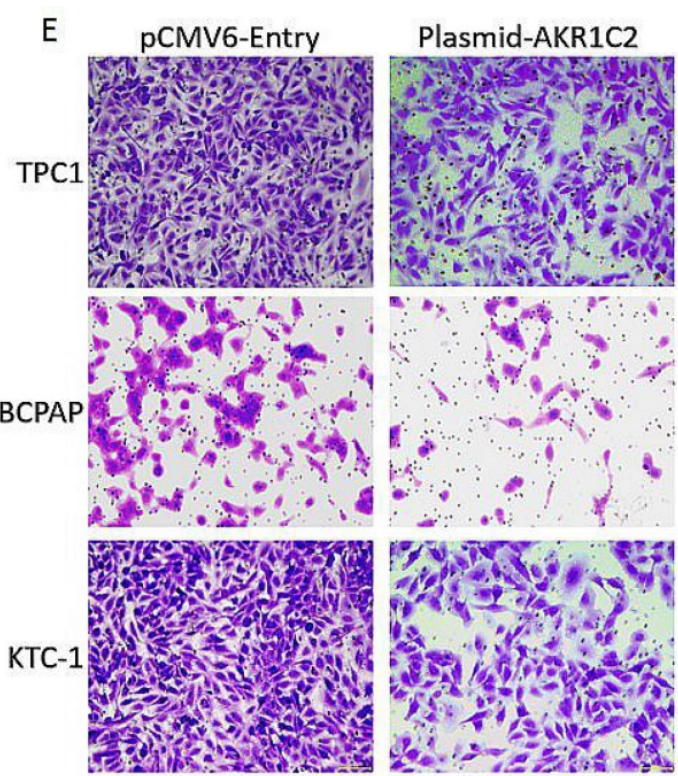

$\mathrm{F}$

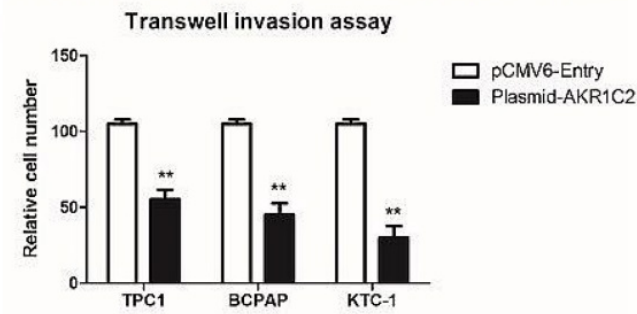

Figure 3. The effect of overexpression of AKR1C2 on cell migration and invasion of TPC-1, BCPAP and KTC-1 thyroid cancer cell lines. Notes (a) qRT-PCR was applied for the confirmation of the overexpression of AKR1C2. Transfected by targeted AKR1C2 cDNA ORF clone, mRNA expression of AKR1C2 was remarkably upregulated in BCPAP, TPC1, and KTC-1 cell lines in contrast to empty control PCMV6-Entry vector. (b) Western blot was used for confirming the overexpression of AKR1C2. Transfected by targeted AKR1C2 cDNA ORF clone, protein expression of AKR1C2 was remarkably upregulated in BCPAP, TPC1, and KTC-1 cell lines in contrast to empty control pCMV6-Entry vector. (c) As for migration assays, we found that compared with the cells transfected by empty control pCMV6-Entry vector, the migrating cells transfected by AKR1C2 cDNA ORF clone were much less in BCPAP, TPC1, and KTC-1 cell lines. (d) The number of the migrating cell by relative quantification. (e): With respect to invasion assays, we found that compared with the cells transfected by empty control PCMV6-Entry vector, the invading cells transfected by AKR1C2 cDNA ORF clone were much less in BCPAP, TPC1, and KTC-1 cell lines. (f) The number of the invading cell relative quantification. The Columns refers to the average number of invading cell from no less than three experiments; and the little vertical bars, which located at the top end of the columns, corresponds to standard deviation (SD). $*<<0.1, * * p<0.05, * * * p<0.01$ using student's t-test.

Table 4. Multivariate cox regression analysis of recurrence-free survival

\begin{tabular}{llll}
\hline Characteristics & HR & $95 \%$ CI & $p$-value \\
\hline AKR1C2 (continuous variable) & 0.819 & $0.677-0.992$ & $0.041^{*}$ \\
AJCC stage & 1.534 & $1.172-2.009$ & $0.002^{*}$
\end{tabular}

${ }^{*} p$-value $<0.05$.

\section{AKR1C2 overexpression inhibits the capacities of migration and invasion in PTC cells}

Furthermore, to validate the role of AKR1C2 in thyroid carcinoma, we upregulated AKR1C2 expression levels using human cDNA ORF clone. We validated the role of AKR1C2 by analyzing the results in ARK1C2 downregulation cohort. In Fig. 3a and 3b, the AKR1C2 expression level is effectively upregulated in all three thyroid cancer cell lines $(p<$ 0.001 ) by preforming qRT-PCR and Western blot,. We also validated the role of AKR1C2 in metastasis. As shown in Fig. $3 \mathrm{c}$ to $3 \mathrm{f}$, Transwell migration and Transwell invasion assays were performed. Results showed that AKR1C2 overexpression significantly weakens the abilities of migration and invasion in thyroid cancer cells TPC-1, BCPAP, and KTC-1 $(p<$ 0.05). 


\section{Discussion}

The incidence of thyroid carcinoma has gradually gone up all over the world in the last several decades, increasing by $4 \%$ annually $[1,19]$. Even though a lot of progress in genomics study has been made, the explicit genetic molecular mechanisms and regulations in PTC remain unclear. Therefore, undiscovered genes relevant to oncogenes is should be discovered immediately.

AKR1C is one of the AKR superfamily members and has four isoforms: AKR1C1, AKR1C2, AKR1C3 and AKR1C4 which share a high degree of homology with each other. Those genes are located on chromosome 10p15.1 and are correlated with the metabolism of steroids [20], prostaglandins [21] and bile acid precursors [22]. Among those genes, AKR1C2 can metabolize progesterone and tobacco carcinogens [23]. Previous studies indicated that upregulated AKR1C2 is associated with platinum drug resistance in cancers [24]. Moreover, AKR1C2 is also related to serum DHT concentration which can lead to obesity [8]. In prostate cancer, this gene can be modulated by curcumin so that could decrease testosterone production and curcumin's natural bioactive compounds could have potent anticancer properties because of suppression of androgen production, and this could have therapeutic effects on prostate cancer [9]. In liver cancer, AKR1C2 is downstream of AGE-1 gene and the later through AKR1C2 promotes metastasis [25].

Currently, with the development of high-throughput sequencing technologies, such as Next-generation sequencing and Microarray, genes differently expressed between tumor and normal tissues have become a focus of research [26]. After analyzing the data downloaded from TCGA database, results showed the differential expression of AKR1C2 between tumor and normal tissues $(p<0.001)$. We then collected a validation group consisting of 38 primary PTCs and matched noncancerous thyroid tissues before measuring AKR1C2 expression. We found that AKR1C2 plays crucial roles in various cancers. Previously, AKR1C2 shows an inhibitory role in the progression of squamous cell carcinomas and breast cancers [12, 14]. Meanwhile, AKR1C2 is a positive regulator in promoting metastasis in liver cancer [15]. In addition, AKR1C2 displays promising potential in anticancer drug resistance [16]. Nevertheless, the connection of AKR1C2 with comprehensive tumorigenesis in thyroid cancer is unknown.

After analyzing the data from TCGA database, we utilized 38 PTC tumor tissues and adjacent normal tissues to explore the role of AKR1C2 in thyroid cancer. The results turned out that AKR1C2 expression is remarkably lower in papillary thyroid carcinoma samples than that in adjacent normal tissues both in validation $(p<0.001)$ and TCGA cohorts $(p<0.001)$. The thyroid cell line experiments showed an inhibitory effect on thyroid cancer metastasis. As shown in Figs. 2 and 3, migration and invasion assays were carried out using targeted si-RNAs and plasmids. The results indicated that the knockdown of AKR1C2 exerts an intensive effect and overexpression of AKR1C2 exerts an inhibitory effect on migration and invasion of PTC cell lines TPC-1, BCPAP, and KTC-1.

According to our knowledge, there were some studies exploring the relationship with RFS, such as lymph node recurrence rate, minimal extrathyroid extension and DNA methylation levels of well-differentiated thyroid [27-29]. However, most of them explore clinicopathologic characteristics with RFS in thyroid carcinoma, a small number of studies explore gene expression with RFS in thyroid cancer [30]. Delightfully, in our study, the univariate Cox regression analysis revealed clinicopathologic characteristics factors highly associated with RFS in Table 3: tumor stage (HR 1.588; 95\% CI, 1.110-2.272; $p$ $=0.011)$, metastasis $(\mathrm{HR}, 8.995 ; 95 \% \mathrm{CI}, 2.596-30.897, p$ $=0.001)$, AJCC stage $(\mathrm{HR}, 1.528 ; 95 \% \mathrm{CI}, 1.167-2.001 ; p$ $=0.002)$, and AKR1C2 expression (HR, 0.824; 95\% CI, $0.681-0.998 ; p=0.047)$. In the next step, Multivariate cox regression analysis of RFS analysis revealed that the factors independently associated with RFS were AKR1C2 expression (HR, 0.819; 95\% CI, 0.677-0.992; $p$ $=0.041)$ and AJCC stage $(\mathrm{HR}, 1.534 ; 95 \% \mathrm{CI}$, 1.172-2.009; $p=0.002$ ) in Table 4. Previous study revealed the relationship between AJCC stage and RFS [31]. As an independent factor associated with RFS, similar to AJCC stage (HR, 1.534; 95\% CI, 1.172-2.009; $p=0.002)$, AKR1C2 expression (HR, $0.819 ; 95 \% \mathrm{CI}, 0.677-0.992 ; p=0.041$ ) was detected significantly downregulated in PTC tumor samples compared to matched adjacent noncancerous tissues. Most importantly, RFS rates, which are the most intuitive method to evaluate the effect of AKR1C2 to thyroid cancer, were observed that over-expressed group had obviously higher RFS rates than under-expressed group in Fig. 1c to 1e. Therefore, given that its expression had HR $<1$ and $p<0.05$ and the expression of AKR1C2 was remarkably less in tumor tissues than in adjacent noncancerous tissues, we would like to assume that AKR1C2 is an independent protective prognosis factor and high expression of AKR1C2 is associated with more favorable prognosis.

Combining the clinicopathological characteristics from TCGA cohort and in vitro experiments, we assumed that high expression of AKR1C2 is likely 
connected with favorable prognosis and low sensitivity to tumorigenesis. In addition, lymph nodes detection might be performed during thyroid surgery due to the high inclination of the under-expressed expression of AKR1C2 to detect lymph node metastasis. And gene AKR1C2 may provide a potential new biomarker in thyroid cancer because of the relationship between AKR1C2 expression and RFS. Most importantly, we might regard gene AKR1C2 as a novel biomarker to evaluate the prognosis, similar to AJCC stage.

Several limitations are also observed. For a more comprehensive study of the specific function and pathway of AKR1C2 in tumors, further relevant experiment (in vitro and in vivo) should be performed in the future. Moreover, we were not able to draw a comprehensive understanding of the specific biological function of AKR1C2. Finally, the regulatory mechanism of AKR1C2 and whether it is involved in changes or epigenetic modifications at the DNA level remain unclear.

In conclusion, our study indicated that (a) AKR1C2 is generally down-expressed in PTC, (b) gene AKR1C2 might exert an inhibitory effect on oncogenesis of PTC, (c) the elevated AKR1C2 are associated with considerably favorable prognostic factors and recurrence free survival in thyroid cancer.

\section{Acknowledgments}

This work was supported by Wenzhou Science and Technology Planning Project (Y20170735), National High Technology Research and Development Program of 863 project of China (NO2012AA02A210), the Major Science Technology Projects of Zhejiang Province (2015C03052) and Scientific Research Incubator Project of The First Affiliated Hospital of Wenzhou Medical University (NO. FHY2014018).

\section{Funding}

This study was funded by Wenzhou Science and Technology Planning Project (Y20170735), National High Technology Research and Development Program of 863 project of China (NO2012AA02A210), the Major Science Technology Projects of Zhejiang Province (2015C03052) and Scientific Research Incubator Project of The First Affiliated Hospital of Wenzhou Medical University (NO. FHY2014018).

\section{Ethical approval}

All applicable international, national, and institutional guidelines for the care were followed. All procedures performed in studies involving human participants were in accordance with the ethical standards of the institutional ethical standards.
Ethical approval for this study was obtained from the Ethnic Committee of the First Affliated Hospital of Wenzhou Medical University.

\section{Informed consent}

Written informed consent was obtained from all individual participant included in the study.

\section{Competing Interests}

The authors have declared that no competing interest exists.

\section{References}

1. Kilfoy BA, Zheng $\mathrm{T}$, Holford TR, Han X, Ward MH, Sjodin A, et al International patterns and trends in thyroid cancer incidence, 1973-2002. Cancer causes \& control : CCC. 2009; 20: 525-31.

2. Pellegriti G, Frasca F, Regalbuto C, Squatrito S, Vigneri R. Worldwide increasing incidence of thyroid cancer: update on epidemiology and risk factors. Journal of cancer epidemiology. 2013; 2013: 965212.

3. Davies L, Welch HG. Current thyroid cancer trends in the United States. JAMA otolaryngology-- head \& neck surgery. 2014; 140: 317-22.

4. Lim H, Devesa SS, Sosa JA, Check D, Kitahara CM. Trends in Thyroid Cancer Incidence and Mortality in the United States, 1974-2013. Jama. 2017; 317: 1338-48.

5. Yang L, Yuan Y, Sun T, Li H, Wang N. Population-based cancer incidence analysis in Beijing, 2008-2012. Chinese journal of cancer research $=$ Chung-kuo yen cheng yen chiu. 2015; 27: 13-21.

6. Markovina S, Grigsby PW, Schwarz JK, DeWees T, Moley JF, Siegel BA, et al. Treatment approach, surveillance, and outcome of well-differentiated thyroid cancer in childhood and adolescence. Thyroid : official journal of the American Thyroid Association. 2014; 24: 1121-6.

7. Cabanillas ME, McFadden DG, Durante C. Thyroid cancer. Lancet (London, England). 2016; 388: 2783-95.

8. Mazzaferri EL, Jhiang SM. Long-term impact of initial surgical and medical therapy on papillary and follicular thyroid cancer. The American journal of medicine. 1994; 97: 418-28.

9. Nikiforov YE, Nikiforova MN. Molecular genetics and diagnosis of thyroid cancer. Nature reviews Endocrinology. 2011; 7: 569-80.

10. Soares P, Celestino R, Melo M, Fonseca E, Sobrinho-Simoes M. Prognostic biomarkers in thyroid cancer. Virchows Archiv : an international journal of pathology. 2014; 464: 333-46.

11. Vu-Phan D, Koenig RJ. Genetics and epigenetics of sporadic thyroid cancer. Molecular and cellular endocrinology. 2014; 386: 55-66.

12. Li W, Hou G, Zhou D, Lou X, Xu Y, Liu S, et al. The roles of AKR1C1 and AKR1C2 in ethyl-3,4-dihydroxybenzoate induced esophageal squamous cell carcinoma cell death. Oncotarget. 2016; 7: 21542-55

13. Mindnich RD, Penning TM. Aldo-keto reductase (AKR) superfamily: genomics and annotation. Human genomics. 2009· 3 362-70.

14. Wenners A, Hartmann F, Jochens A, Roemer AM, Alkatout I, Klapper W, et al. Stromal markers AKR1C1 and AKR1C2 are prognostic factors in primary human breast cancer. Int J Clin Oncol. 2016; 21: 548-56.

15. Li C, Wu X, Zhang W, Li J, Liu H, Hao M, et al. High-Content Functional Screening of AEG-1 and AKR1C2 for the Promotion of Metastasis in Liver Cancer. Journal of biomolecular screening. 2016; 21: 101-7.

16. Shiiba M, Yamagami H, Yamamoto A, Minakawa Y, Okamoto A, Kasamatsu A, et al. Mefenamic acid enhances anticancer drug sensitivity via inhibition of aldo-keto reductase 1C enzyme activity. Oncology reports. 2017; 37: 2025-32.

17. Huang $\mathrm{KH}$, Chiou $\mathrm{SH}$, Chow $\mathrm{KC}$, Lin TY, Chang HW, Chiang IP, et al. Overexpression of aldo-keto reductase 1C2 is associated with disease progression in patients with prostatic cancer. Histopathology. 2010; 57: 384-94.

18. Cancer Genome Atlas Research Network. Integrated genomic characterization of papillary thyroid carcinoma. Cell. 2014; 159: 676-90.

19. Li Z, Huang X, Xu J, Su Q, Zhao J, Ma J. miR-449 overexpression inhibits papillary thyroid carcinoma cell growth by targeting RET kinase-beta-catenin signaling pathway. International journal of oncology. 2016; 49: 1629-37.

20. Schlegel BP, Pawlowski JE, Hu Y, Scolnick DM, Covey DF, Penning TM. Secosteroid mechanism-based inactivators and site-directed mutagenesis as probes for steroid hormone recognition by 3 alpha-hydroxysteroid dehydrogenase. Biochemistry. 1994; 33: 10367-74.

21. Penning TM. The aldo-keto reductases (AKRs): Overview. Chemico-biological interactions. 2015; 234: 236-46.

22. Hyndman D, Bauman DR, Heredia VV, Penning TM. The aldo-keto reductase superfamily homepage. Chemico-biological interactions. 2003; 143-144: 621-31.

23. Stapelfeld C, Neumann KT, Maser E. Different inhibitory potential of sex hormones on NNK detoxification in vitro: A possible explanation for gender-specific lung cancer risk. Cancer letters. 2017; 405: 120-6.

24. Vihma V, Naukkarinen J, Turpeinen U, Hamalainen E, Kaprio J, Rissanen A, et al. Metabolism of sex steroids is influenced by acquired adiposity-A study of 
young adult male monozygotic twin pairs. The Journal of steroid biochemistry and molecular biology. 2017; 172: 98-105.

25. Li C, Wu X, Zhang W, Li J, Liu H, Hao M, et al. AEG-1 Promotes Metastasis Through Downstream AKR1C2 and NF1 in Liver Cancer. Oncology research. 2014; 22: 203-11.

26. Zheng Y, Qing T, Song Y, Zhu J, Yu Y, Shi W, et al. Standardization efforts enabling next-generation sequencing and microarray based biomarkers for precision medicine. Biomarkers in medicine. 2015; 9: 1265-72.

27. Ito $\mathrm{Y}$, Kudo T, Kihara M, Takamura Y, Kobayashi K, Miya A, et al. Improvement of lymph node recurrence rate, but not distant recurrence and carcinoma death rates, in patients with papillary thyroid carcinoma after disease-free survival for 5 years. Endocrine journal. 2012; 59: 895-901.

28. Woo CG, Sung CO, Choi YM, Kim WG, Kim TY, Shong YK, et al. Clinicopathological Significance of Minimal Extrathyroid Extension in Solitary Papillary Thyroid Carcinomas. Annals of surgical oncology. 2015; 22 Suppl 3: S728-33.

29. Mancikova V, Buj R, Castelblanco E, Inglada-Perez L, Diez A, de Cubas AA, et al. DNA methylation profiling of well-differentiated thyroid cancer uncovers markers of recurrence free survival. International journal of cancer. 2014; 135: 598-610.

30. Choi JY, Yi JW, Lee JH, Song RY, Yu H, Kwon H, et al. VDR mRNA overexpression is associated with worse prognostic factors in papillary thyroid carcinoma. Endocrine connections. 2017; 6: 172-8.

31. Wang LY, Nixon IJ, Palmer FL, Thomas D, Tuttle RM, Shaha AR, et al. Comparable outcomes for patients with $\mathrm{pT} 1 \mathrm{a}$ and $\mathrm{pT} 1 \mathrm{~b}$ differentiated thyroid cancer: Is there a need for change in the AJCC classification system? Surgery. 2014; 156: 1484-9; discussion 9-90. 